\title{
D-state configurations in the electromagnetic form factors of the nucleon and the $\Delta(1232)$ resonance
}

\author{
B. Juliá-Díaz ${ }^{\mathrm{a}, \mathrm{b}}$ D.O. Riska ${ }^{\mathrm{b}}$ \\ ${ }^{a}$ Department of Physics and Astronomy, University of Pittsburgh, PA 15260, USA \\ ${ }^{\mathrm{b}}$ Helsinki Institute of Physics and Department of Physical Sciences, P.O.Box 64, \\ 00014 University of Helsinki, Finland
}

\begin{abstract}
The $\Delta-N$ electromagnetic transition form factors are calculated in the Poincaré covariant quark model in three forms of relativistic kinematics. Addition of $D$-state components to pure $S$-state model wave functions, chosen so as to reproduce the empirical elastic electromagnetic nucleon form factors with single constituent currents, brings the calculated $R_{E M}$ ratio for the $\Delta(1232) \rightarrow N \gamma$ transition closer to the empirical values in instant and point form kinematics. The calculated $R_{S M}$ ratio is insensitive to the $D$-state component. In front form kinematics the substantial violation of the angular condition for the spin $3 / 2$ resonance transition amplitude in the impulse approximation prevents a unique determination of $R_{E M}$ and $R_{S M}$, both of which are very sensitive to $D$-state components. In no form of kinematics do $D$-state deformations of the rest frame baryon wave functions alone suffice for a description of the empirical values of these ratios.
\end{abstract}

\section{Introduction}

Deformation of the nucleon wave function from spherical symmetry in the rest frame, and its possible empirical manifestations have drawn considerable experimental and theoretical attention $[1,2,3,4]$. The experimental finding of nonvanishing $E 2 / M 1$ and $C 2 / M 1$ ratios in the electromagnetic decay of the $\Delta(1232)$ suggests the presence either of spatial $D$-state components in the wave functions or of quark-antiquark configurations ("meson cloud effects $[5,6] ")$.

The elastic form factors of the proton and the magnetic form factor of the neutron may be described in the constituent quark model, with point-like quarks and spherically symmetric $S$-state rest frame wave functions in all the

Preprint submitted to Elsevier Science 
three forms of relativistic kinematics outlined by Dirac [7]. With an additional small $(\sim 1 \%)$ admixture of a mixed symmetry S-state component the electric form factor of the neutron may be described as well [8]. The ranges of the wave functions do however differ significantly between the different forms of kinematics, as do the high- $Q^{2}$ behaviors of the form factors.

In the covariant quark model both the electric form factor of the nucleon and the $E 2 / M 1$ and $C 2 / M 1$ ratios in $\Delta \rightarrow N \gamma$ decay are non-zero even in the case of spatially symmetric $S$-state three-quark wave functions in the rest frame [9]. This is a consequence of the kinematic boosts and the Wigner rotations (or Melosh rotations in the case of front form kinematics), which are absent in the non-relativistic quark model. The key question then becomes to what extent admixtures of components with higher angular momenta or of quark-antiquark components may be required in the baryon wave functions for a satisfactory description of the empirical observables.

It is shown here that the effect of adding $D$-state components to the rest frame wave functions of the proton and the $\Delta(1232)$ resonance is significant for the $E 2 / M 1$ ratio $R_{E M}$, but very small for the $C 2 / M 1$ ratio $R_{S M}$ in the case of instant and point form kinematics. It is found that with single quark currents the notable empirical (negative) peak in $R_{E M}$ below $0.5 \mathrm{GeV}^{2}$ cannot be described by $D$-state deformations alone. It is also found that the covariant quark model yields small positive values for $R_{S M}$, in disagreement with the empirically found negative values.

In the case of the $\Delta(1232) \rightarrow N \gamma$ transition, front form kinematics does not yield unique results for the $E 2 / M 1$ and $C 2 / M 1$ ratios, because of the violation of the linear relation between more than three different spin amplitudes in the front form description of form factors of states with spin $3 / 2$ and single constituent current operators $[10,11,12]$. The calculated magnetic $\Delta(1232) \rightarrow N \gamma$ transition form factor is, however, insensitive to this problem, and is qualitatively similar to that obtained in instant and front form kinematics.

The article is organized as follows. In Section 2 the calculation of the transition form factors from the matrix elements of the electromagnetic current of the constituent quarks is outlined for all the three forms of kinematics. In Section 3 the explicit baryon wave function model that includes $D$-state components is described. In Section 4 the matrix elements required for the calculation of the $\Delta(1232) \rightarrow N \gamma$ transition are given explicitly. In Section 5 the effects of the $D$-state components are studied in detail. A concluding summary is provided in the last section. 


\section{$2 \Delta(1232)-\mathrm{N} \gamma$ transition form factors}

The electromagnetic elastic and transition form factors of the baryons are linear combinations of matrix elements of the electromagnetic current. In instant and point form kinematics the appropriate matrix elements for the $\Delta(1232) \rightarrow N \gamma$ transition form factors are matrix elements of the $I_{1}$ and $I_{0}$ components of the current operator.

The general expression for the transition current operator:

$$
\left\langle\Delta\left|\mathcal{I}^{\mu}(0)\right| N\right\rangle=e \bar{\Psi}_{\nu}\left(p^{*}\right) \Gamma^{\nu \mu} \Psi(p),
$$

has the conventional decomposition [8]:

$$
\Gamma^{\nu \mu}=\sum_{i} G_{i}\left(Q^{2}\right) \mathcal{K}_{i}^{\nu \mu} .
$$

The operators $\mathcal{K}_{i}^{\nu \mu}$ are defined as:

$$
\begin{aligned}
\mathcal{K}_{1}^{\nu \mu} & =\frac{Q^{\nu} \gamma^{\mu}-(\gamma \cdot Q) g^{\nu \mu}}{\sqrt{Q^{2}}} \sqrt{M^{*} M} \gamma_{5}, \\
\mathcal{K}_{2}^{\nu \mu} & =\frac{Q^{\mu} P^{\nu}-(P \cdot Q) g^{\nu \mu}}{\sqrt{Q^{2}}} \gamma_{5}, \\
\mathcal{K}_{3}^{\nu \mu} & =\frac{Q^{\nu} Q^{\mu}-Q^{2} g^{\nu \mu}}{\sqrt{Q^{2}}} M^{*} \gamma_{5} .
\end{aligned}
$$

Here $M$ and $M^{*}$ are the masses of the nucleon and $\Delta(1232)$. The relation between the three form factors $G_{1}, G_{2}$ and $G_{3}$ and the corresponding electric, magnetic and Coulomb form factors are [13]:

$$
\begin{aligned}
G_{E}^{*} & =\frac{M}{3\left(M^{*}+M\right)}\left[\frac{M^{* 2}-M^{2}-Q^{2}}{M^{*}} \frac{\sqrt{M^{*} M}}{Q} G_{1}\right. \\
& \left.+\frac{M^{* 2}-M^{2}}{Q} G_{2}-2 M^{*} G_{3}\right], \\
G_{M}^{*} & =\frac{M}{3\left(M^{*}+M\right)}\left[\frac{\left(3 M^{*}+M\right)\left(M^{*}+M\right)+Q^{2}}{M^{*}} \frac{\sqrt{M^{*} M}}{Q} G_{1}\right. \\
& \left.+\frac{M^{* 2}-M^{2}}{Q} G_{2}-2 M^{*} G_{3}\right], \\
G_{C}^{*} & =\frac{2 M}{3\left(M^{*}+M\right)}\left[\frac{2 M^{*} \sqrt{M^{*} M}}{Q} G_{1}+\frac{3 M^{* 2}+M^{2}+Q^{2}}{2 Q} G_{2}\right.
\end{aligned}
$$




$$
\left.+\frac{M^{* 2}-M^{2}-Q^{2}}{Q^{2}} M^{*} G_{3}\right] .
$$

In instant and point form kinematics the relation between the different spin state matrix elements of the electromagnetic current and the form factors, $G_{j}$, is finally:

$$
\begin{aligned}
I_{\frac{3}{2}, \frac{1}{2}}^{1} & =\left[\frac{M^{*}+M}{\sqrt{Q^{2}}} G_{1}+\frac{M^{* 2}-M^{2}}{2 \sqrt{Q^{2} M M^{*}}} G_{2}-\sqrt{\frac{M^{*}}{M}} G_{3}\right] \frac{Q_{3}}{2 \sqrt{E(M+E)}} \\
I_{\frac{1}{2},-\frac{1}{2}}^{1} & =-\frac{\sqrt{3}}{6}\left[\frac{M^{*}+M}{\sqrt{Q^{2}}} G_{1}+\frac{M^{* 2}-M^{2}}{2 \sqrt{Q^{2} M M^{*}}} G_{2}-\sqrt{\frac{M^{*}}{M}} G_{3}\right] \frac{Q_{3}}{\sqrt{E(M+E)}} \\
& +\frac{\sqrt{3}}{3} \frac{Q_{3}}{\sqrt{Q^{2}}} \frac{M+E}{\sqrt{E(M+E)}} G_{1}, \\
I_{\frac{1}{2}, \frac{1}{2}}^{0} & =-\frac{\sqrt{3}}{3}\left[\frac{Q_{3}}{\sqrt{Q^{2}}} G_{1}+\frac{Q_{3}}{\sqrt{Q^{2} M M^{*}}} \frac{E+M^{*}}{2} G_{2}\right. \\
& \left.+\frac{Q_{3} Q_{0}}{Q^{2}} \sqrt{\frac{M^{*}}{M}} G_{3}\right] \frac{Q_{3}}{\sqrt{E(M+E)}} .
\end{aligned}
$$

Here the 4-momentum transfer is taken as $Q=\left\{Q^{0}, 0,0, Q_{3}\right\}$, with $I_{j_{\Delta}, j_{N}}^{m}=$ $\left\langle j_{\Delta}, P_{\Delta}\left|I_{m}(0)\right| P_{N}, j_{N}\right\rangle$, and

$$
Q^{0}=-\frac{P^{*} \cdot Q}{M^{*}}=\frac{M^{* 2}-M^{2}-Q^{2}}{2 M^{*}}, \quad Q_{3}=\sqrt{Q^{2}+Q^{02}} .
$$

The $E 2 / M 1$ and $C 2 / M 1$ ratios for the $\Delta-N$ transition are defined as ${ }^{1}$ :

$$
\begin{aligned}
R_{E M} & \equiv \frac{E_{2}}{M_{1}} \equiv-\frac{G_{E}^{*}}{G_{M}^{*}}, \\
R_{S M} & \equiv \frac{C_{2}}{M_{1}} \equiv \frac{|\vec{q}|}{2 M^{*}} \frac{G_{C}^{*}}{G_{M}^{*}} .
\end{aligned}
$$

Here $2 M^{*}|\vec{q}|=\left(\left[Q^{2}+\left(M^{*}-M\right)^{2}\right]\left[Q^{2}+\left(M^{*}+M\right)^{2}\right]\right)^{1 / 2}$.

In the application of front form kinematics it has been conventional to adopt a reference frame in which $Q^{+}=0$. In the case of elastic form factors the relation between the initial and final state is kinematic in this frame ${ }^{2}$. The relation

$\overline{1}$ The definition of $R_{S M}$ is that of Ref. [6], the overall sign of which disagrees with that in Ref. [11].

2 These relations together with Eq. (4) are equivalent to the definition of Eq.(3) of [11] together with the form factors of Ref. [14]. 
between the invariant form factors $G_{j}$ and the current matrix elements in this frame is

$$
\begin{aligned}
I_{\frac{3}{2}, \frac{1}{2}} & =\frac{1}{\sqrt{2}}\left[G_{1}+\frac{M_{\Delta}-M}{\sqrt{4 M M_{\Delta}}} G_{2}\right], \\
I_{\frac{1}{2}, \frac{1}{2}} & =-\frac{1}{\sqrt{6}}\left[Q \frac{G_{1}}{M_{\Delta}}+Q \frac{2 M_{\Delta}-M}{M_{\Delta} \sqrt{4 M M_{\Delta}}} G_{2}-\frac{M_{\Delta}-M}{\sqrt{M M_{\Delta}}} G_{3}\right], \\
I_{\frac{1}{2},-\frac{1}{2}} & =-\frac{1}{\sqrt{6}}\left[-\frac{M}{M_{\Delta}} G_{1}+\frac{M_{\Delta}\left(M_{\Delta}-M\right)-Q^{2}}{M_{\Delta} \sqrt{4 M M_{\Delta}}} G_{2}+\frac{Q}{\sqrt{M M_{\Delta}}} G_{3}\right], \\
I_{\frac{3}{2},-\frac{1}{2}} & =-\frac{Q}{2 \sqrt{2 M M_{\Delta}}} G_{2} .
\end{aligned}
$$

Here $I_{\nu_{1}, \nu_{2}}$ is defined as:

$$
I_{\nu_{1}, \nu_{2}}=\left\langle\Delta, \nu_{1}\left|\mathcal{I}^{+}\right| N, \nu_{2}\right\rangle
$$

where $\mathcal{I}^{+}=n \cdot \mathcal{I}$ and $n$ is the null-vector: $n=\{-1,0,0,1\}$.

These four spin amplitudes are linear combinations of only three invariant transition form factors and therefore they must are linearly dependent. This linear relation is broken by the sum of matrix elements of single quark currents in front form kinematics, and as a consequence, the calculated transition form factors depend on which set of three spin amplitudes are employed to calculate the transition form factors [11,12]. This prevents definite predictions in front form kinematics for form factors of states with spin larger than $1 / 2$ when only single quark currents are employed.

In the case of the transition form factors the $Q^{+}=0$ frame is not required by the kinematics, and has the disadvantage of requiring analytic extrapolation to the region of timelike momenta. For transition form factors it may be more appropriate to adopt a frame in which $Q^{+} \neq 0$ and which allows extrapolation to timelike momenta with real kinematic coefficients [15].

This frame may be defined as in Ref. [15]:

$$
v_{f}=\{\sqrt{1+\eta}, \sqrt{\eta}, 0,0\}, \quad v_{a}=\{\sqrt{1+\eta},-\sqrt{\eta}, 0,0\},
$$

so that

$$
\begin{aligned}
& Q^{+}=\left(M_{f}-M_{a}\right) \sqrt{1+\eta}, \quad Q_{\perp}=\left(M_{f}+M_{a}\right) \sqrt{\eta}, \\
& M_{a} p_{f}^{+}=M_{f} p_{a}^{+}, \quad M_{a} p_{f \perp}+M_{f} p_{a \perp}=0 .
\end{aligned}
$$


In this frame the relation between the invariant form factors $G_{j}$ and the current matrix elements are:

$$
\begin{aligned}
I_{\frac{3}{2}, \frac{1}{2}} & =-\frac{\sqrt{2} M_{N} \sqrt{\eta}}{Q} G_{1}, \\
I_{\frac{1}{2}, \frac{1}{2}} & =\sqrt{\frac{M_{N}}{M_{\Delta}}} \frac{\eta}{\sqrt{6}}\left[4 \sqrt{\frac{M_{\Delta} M_{N}}{Q^{2}}} G_{1}+\frac{M_{\Delta}+M_{N}}{Q} G_{2}\right. \\
& \left.+\frac{2 M_{\Delta}\left(M_{\Delta}-M_{N}\right)}{Q^{2}} G_{3}\right], \\
I_{\frac{1}{2},-\frac{1}{2}} & =-\frac{\sqrt{\eta}}{\sqrt{6}}\left[2 \frac{M_{\Delta}}{Q} G_{1}+\frac{M_{\Delta}^{2}-M_{N}^{2}+2 \eta M_{N}\left(M_{\Delta}+M_{N}\right)}{Q \sqrt{M_{N} M_{\Delta}}} G_{2}\right. \\
& \left.+2 \sqrt{\frac{M_{\Delta}}{M_{N}}} \frac{2 \eta M_{N}\left(M_{\Delta}-M_{N}\right)-Q^{2}}{Q^{2}} G_{3}\right], \\
I_{\frac{3}{2},-\frac{1}{2}} & =\sqrt{\frac{M_{N}}{M_{\Delta}}} \frac{\eta}{\sqrt{2}}\left[\frac{M_{\Delta}+M_{N}}{Q} G_{2}+\frac{2 M_{\Delta}\left(M_{\Delta}-M_{N}\right)}{Q^{2}} G_{3}\right] .
\end{aligned}
$$

In this case the last one of these spin amplitudes is a linear combination of the first two. This "angular condition" is broken by the matrix elements of the sum of single quark current operators in front form kinematics, and therefore the calculated transition form factors also in this case depend on which set of three amplitudes are used to determine the three invariant transition form factors.

\section{The nucleon and $\Delta(1232)$ wave functions}

The rest frame wave function of the nucleon shall be taken to be a combination of $S$ - and $D$-state components as:

$$
\phi_{N}=a_{N} \phi_{S}+b_{N} \phi_{D}
$$

where $\left\langle\phi_{S} \mid \phi_{S}\right\rangle=1,\left\langle\phi_{S} \mid \phi_{D}\right\rangle=0$ and $\left\langle\phi_{D} \mid \phi_{D}\right\rangle=1$ so that the sum of the amplitudes squared is unity: $\left|a_{N}\right|^{2}+\left|b_{N}\right|^{2}=1$. The two components of the wave function are taken to have the following forms, respectively:

$$
\begin{aligned}
& \phi_{S}^{j_{3}}=\varphi_{0}(\mathrm{P}) \chi_{S F}^{S ; j_{3}} \\
& \phi_{D}^{j_{3}}=\frac{1}{\sqrt{2}} \sum_{m s}\left(2 \frac{3}{2} m s \mid \frac{1}{2} j_{3}\right)\left\{\kappa^{2} Y_{2 m}(\hat{\kappa}) \chi_{F}^{M S}+q^{2} Y_{2 m}(\hat{q}) \chi_{F}^{M A}\right\} \varphi_{2}(\mathrm{P}) \chi_{S}^{S ; s}
\end{aligned}
$$


The spatial $S$ - and $D$-state wave functions $\varphi_{0}(\mathrm{P})$ and $\varphi_{2}(\mathrm{P})$ are functions of the hyperspherical momentum variable $\mathrm{P}=\sqrt{2\left(\vec{\kappa}^{2}+\vec{q}^{2}\right)}$, where $\vec{\kappa}$ and $\vec{q}$ are the Jacobi momenta of the 3-quark system. Here the symmetric spin-flavor wave function $\chi_{S F}^{S ; j_{3}}$ is defined as:

$$
\chi_{S F}^{S ; j_{3}}=\frac{1}{\sqrt{2}}\left[\chi_{F}^{M S} \chi_{S}^{M S}+\chi_{F}^{M A} \chi_{S}^{M A}\right]
$$

where $\chi_{F(S)}^{M S}$ is a mixed symmetry flavor (spin) wave function, and $\chi_{F(S)}^{M A}$ is a mixed antisymmetric flavor (spin) wave function. The function $\chi_{S}^{S ; s}$ is a spin-3/2 spin-function.

The explicit expressions for the spatial wave functions are taken to be

$$
\begin{aligned}
& \varphi_{0}(\mathrm{P})=\mathcal{N}_{0}\left(1+\frac{\mathrm{P}^{2}}{4 b^{2}}\right)^{-a}, \\
& \varphi_{2}(\mathrm{P})=\mathcal{N}_{2} \frac{b^{4}}{\mathrm{P}^{2}}\left(\varphi_{0}^{\prime \prime}(\mathrm{P})-\frac{1}{\mathrm{P}} \varphi_{0}^{\prime}(\mathrm{P})\right)^{2} .
\end{aligned}
$$

$\mathcal{N}_{0}$ and $\mathcal{N}_{2}$ are normalization constants and $a$ and $b$ are parameters. The form of the $D$-state wave function $\varphi_{2}(\mathrm{P})$ is chosen so that it has the appropriate threshold behavior in the hyperspherical representation at small distances.

The parameter choices, which lead to a satisfactory description of the electric form factor of the nucleon with Dirac quark currents without the $D$-state component, are $a=6, b=600 \mathrm{MeV}$ in instant and $a=9 / 4, b=640 \mathrm{MeV}$ in point form kinematics [8]. For front form kinematics the corresponding parameter choices are $a=4$ and $b=500 \mathrm{MeV}$.

The wave function of the $\Delta(1232)$ resonance is taken to be formed of $S-$ and $D$-state components in a corresponding way as (cf. Eq. (13):

$$
\phi_{\Delta}=a_{\Delta} \phi_{S}^{\Delta}+b_{\Delta} \phi_{D}^{\Delta},
$$

where $\left\langle\phi_{S}^{\Delta} \mid \phi_{S}^{\Delta}\right\rangle=1,\left\langle\phi_{S}^{\Delta} \mid \phi_{D}^{\Delta}\right\rangle=0$ and $\left\langle\phi_{D}^{\Delta} \mid \phi_{D}^{\Delta}\right\rangle=1$, so that $\left|a_{\Delta}\right|^{2}+\left|b_{\Delta}\right|^{2}=1$.

The explicit expressions for the two components of the $\Delta(1232)$ wave function are:

$$
\begin{aligned}
\phi_{S}^{\Delta ; s_{3}}= & \varphi_{0}(\mathrm{P}) \chi_{S}^{s_{3}} \chi_{F}^{3 / 2}, \\
\phi_{D}^{\Delta ; j_{3}}= & \frac{1}{\sqrt{2}} \sum_{m s}\left(2 \frac{1}{2} m s \mid \frac{3}{2} j_{3}\right)\left\{\kappa^{2} Y_{2 m}(\hat{\kappa}) \chi_{S}^{M S, s}+q^{2} Y_{2 m}(\hat{q}) \chi_{S}^{M A, s}\right\} \\
& \varphi_{2}(\mathrm{P}) \chi_{F}^{3 / 2 ; T} .
\end{aligned}
$$




\section{$4 \quad \Delta(1232)-\mathrm{N}$ matrix elements}

The calculation of the matrix elements of the single quark current operators in the Breit frame, with account of the Lorentz boosts from the initial and final baryon rest frames and the associated Wigner rotations may be carried out using the formalism given in Refs. [8,15].

The matrix element of the current can be written schematically as:

$$
\left\langle\phi_{\Delta}^{j_{3}^{\prime}}|\mathcal{X} S F| \phi_{N}^{j_{3}}\right\rangle=\left(a_{\Delta} \phi_{S}^{\Delta ; j_{3}^{\prime}}+b_{\Delta} \phi_{D}^{\Delta ; j_{3}^{\prime}}\right) \mathcal{X} S F\left(a_{N} \phi_{S}^{N ; j_{3}}+b_{N} \phi_{D}^{N ; j_{3}}\right) .
$$

Here $\mathcal{X}$ is a spatial, $\mathcal{S}$ a spin and $\mathcal{F}$ a flavor operator.

The flavor matrix elements for the $\Delta(1232)^{+} \rightarrow N$ transition are:

$$
\left\langle\chi_{F}^{S}|\mathcal{F}| \chi_{F}^{M S}\right\rangle=\frac{2}{3 \sqrt{2}}, \quad\left\langle\chi_{F}^{S}|\mathcal{F}| \chi_{F}^{M A}\right\rangle=0 .
$$

Given these the calculation reduces to the evaluation of the following matrix elements of spin and spatial operators:

$$
\begin{aligned}
& \left\langle\phi_{S}^{\Delta ; j_{3}^{\prime}}|\mathcal{X} S F| \phi_{S}^{N ; j_{3}}\right\rangle=\frac{1}{3}\left\langle\varphi_{0}|\mathcal{X}| \varphi_{0}\right\rangle\left\langle\chi_{S}^{j_{3}^{\prime}}|\mathcal{S}| \chi_{S}^{M S ; j_{3}}\right\rangle, \\
& \left\langle\phi_{S}^{\Delta ; j_{3}^{\prime}}|\mathcal{X} S F| \phi_{D}^{N ; j_{3}}\right\rangle=\frac{1}{3} \sum_{m s}\left(2 \frac{3}{2} m s \mid \frac{1}{2} j_{3}\right)\left\langle\varphi_{0}|\mathcal{X}| O_{m}^{S}\right\rangle\left\langle\chi_{S}^{j_{3}^{\prime}}|\mathcal{S}| \chi_{S}^{S ; s}\right\rangle, \\
& \left\langle\phi_{D}^{\Delta ; j_{3}^{\prime}}|\mathcal{X} S F| \phi_{S}^{N ; j_{3}}\right\rangle=\frac{1}{3 \sqrt{2}} \sum_{m^{\prime} s^{\prime}}\left(2 \frac{1}{2} m^{\prime} s^{\prime} \mid \frac{3}{2} j_{3}^{\prime}\right) \\
& \left(\left\langle O_{m^{\prime}}^{S}|\mathcal{X}| \varphi_{0}\right\rangle\left\langle\chi_{S}^{M S ; s^{\prime}}|\mathcal{S}| \chi_{S}^{M S ; j_{3}}\right\rangle+\left\langle O_{m^{\prime}}^{A}|\mathcal{X}| \varphi_{0}\right\rangle\left\langle\chi_{S}^{M A ; s^{\prime}}|\mathcal{S}| \chi_{S}^{M S ; j_{3}}\right\rangle\right), \\
& \left\langle\phi_{D}^{\Delta ; j_{3}^{\prime}}|\mathcal{X} S F| \phi_{D}^{N ; j_{3}}\right\rangle=\frac{1}{3 \sqrt{2}} \sum_{m^{\prime} s^{\prime}}\left(2 \frac{1}{2} m^{\prime} s^{\prime} \mid \frac{3}{2} j_{3}^{\prime}\right) \sum_{m s}\left(2 \frac{3}{2} m s \mid \frac{1}{2} j_{3}\right) \\
& \left\{\left\langle O_{m^{\prime}}^{S}|\mathcal{X}| O_{m}^{S}\right\rangle\left\langle\chi_{S}^{M S ; s^{\prime}}|\mathcal{S}| \chi_{S}^{S ; s}\right\rangle+\left\langle O_{m^{\prime}}^{A}|\mathcal{X}| O_{m}^{S}\right\rangle\left\langle\chi_{S}^{M A ; s^{\prime}}|\mathcal{S}| \chi_{S}^{S ; s}\right\rangle\right\} .
\end{aligned}
$$

\section{Numerical signatures of D-state admixtures}

\subsection{The elastic nucleon form factors}

The calculated electric and magnetic form factors of the proton and the mag-

netic form factor of the neutron as obtained in instant, point and front form 

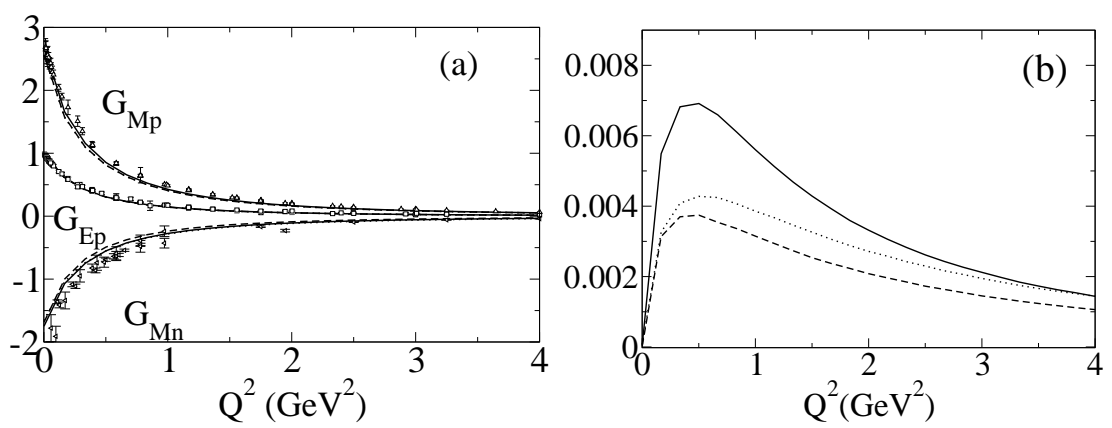

Fig. 1. Electric and magnetic form factors of the proton (a) and the neutron (b) calculated with the $D$-state amplitude $b_{N}=0$ (solid), $b_{N}=0.2$ (dotted) and $b_{N}=-0.2$ (dashed) in instant form kinematics. The experimental data are from the compilation of Ref. [16].

kinematics are shown in Figs. 1(a), 2(a) and 3(a). The results are given with the $D$-state amplitude $b_{N}$ taken to be $0,0.2$ and -0.2 respectively. It is apparent that the effect of a small $D$-state component is almost negligible for both the magnetic form factors as well as for the electric form factor of the proton.

In the (b)-part of the same figures the calculated electric form factor of the neutron is shown for the admixtures $b_{N}$. In the case of $G_{E n}$ the description of the empirical form factor values deteriorates with increasing $D$-state amplitude for the case of instant and point forms. In the case of front form kinematics the calculated move towards the empirical values, when $b_{N}$ is negative. The effects of the $D$-state component are nonetheless very small as compared to that of a small admixture of mixed symmetry $S$-state component [8].
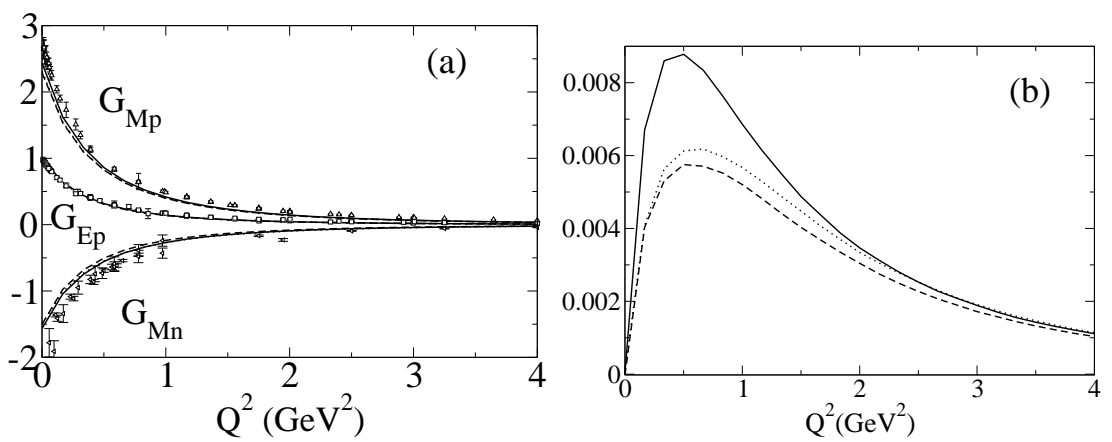

Fig. 2. Same as Fig. 1 but with point form kinematics. 

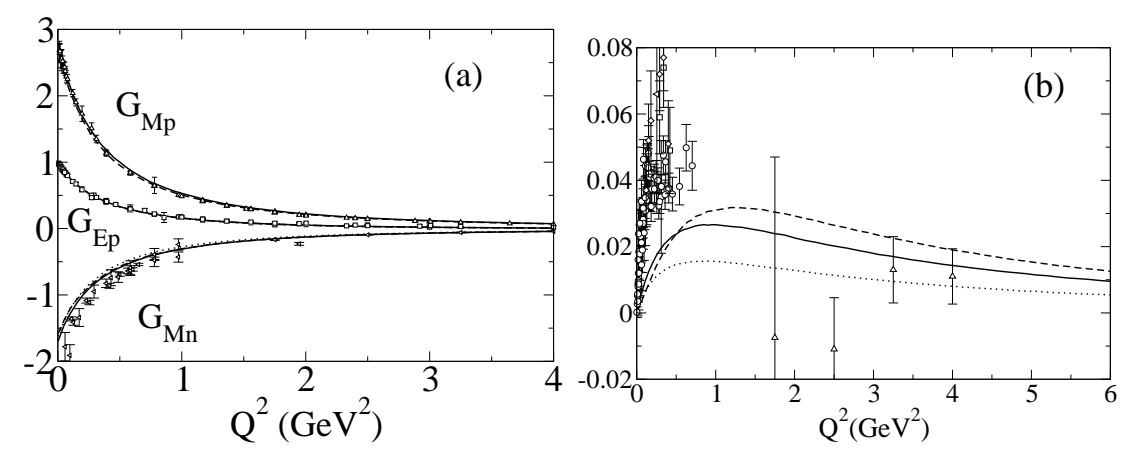

Fig. 3. Same as Fig. 1 but for front form kinematics.
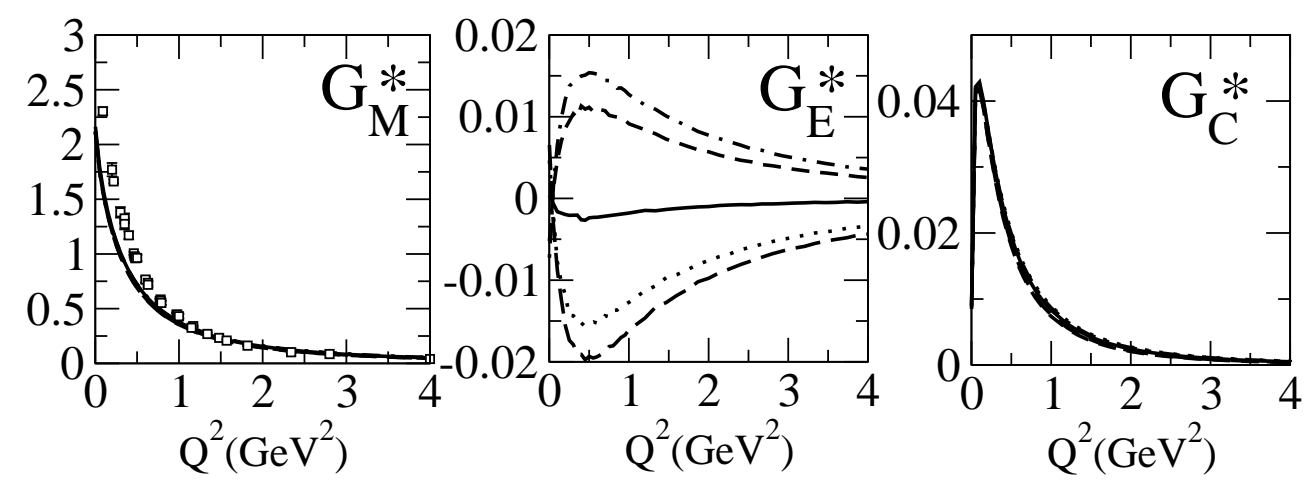

Fig. 4. $G_{E}^{*}, G_{M}^{*}$ and $G_{C}^{*}$ obtained in instant form. Solid, dotted, dashed, long-dashed and dot-dashed lines stand for $\left(b_{N}, b_{\Delta}\right)=(0,0),(0.2,0),(-0.2,0),(0,0.2)$ and $(0,-0.2)$ respectively. In the case of $G_{M}^{*}$ and $G_{C}^{*}$ the lines are almost coincident. The data points for $G_{M}^{*}$ are from Ref. [17].

\subsection{The $\Delta(1232) \rightarrow-N \gamma$ transition form factors}

\subsubsection{Instant form kinematics}

The three transition form factors $G_{M}^{*}, G_{E}^{*}$ and $G_{C}^{*}$ are shown as calculated in instant form kinematics in Fig. 4 for the following combinations of the $D$-state component amplitudes in the nucleon and the $\Delta(1232)$ wave functions: $\left(b_{N}, b_{\Delta}\right)=(0,0),(0.2,0),(-0.2,0),(0,0.2)$ and $(0,-0.2)$. The results reveal that the presence of a $D$-state component only notably affects the electric transition form factor $G_{E}^{*}$. They also reveal that the $D$-state components in the nucleon and the $\Delta(1232)$ have very similar signatures.

In Fig. 5 the corresponding calculated values of the ratios $R_{E M}$ and $R_{S M}$ are shown. The result shows that the effect of the presence of a small $D$-state 
component is very small on the latter ratio. In the case of the $E 2 / M 1$ ratio $R_{E M}$ the addition of the small $D$-state component does in contrast lead to a change in sign of the calculated value for the case of $b_{N(\Delta)}<0$, so that the calculated value takes the same sign the empirical values [18] for $Q^{2} \geq 0.5$ $\mathrm{GeV}^{2}$. The notable empirical structure at lower values of invariant momentum transfer is however not described by $D$-state configurations alone. The extant data are far from the asymptotic behavior $R_{E M} \rightarrow 1$ for $Q^{2} \rightarrow \infty$ suggested by perturbative QCD $[19,20]$.

In the case of the ratio $R_{S M}$ the quark model results are not brought any closer towards the empirical values [18] by introduction of $D$-state components.
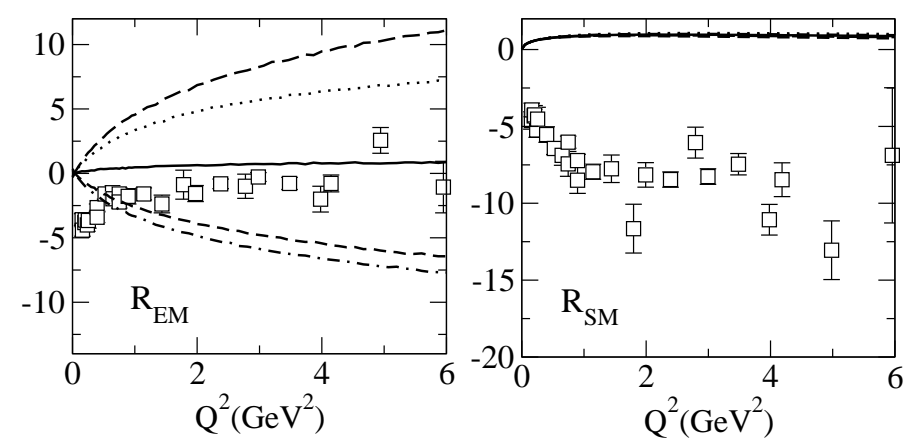

Fig. 5. $R_{E M}$ and $R_{S M}$ ratios in percent obtained in instant form. Solid, dotted, dashed, long-dashed and dot-dashed lines stand for $\left(b_{N}, b_{\Delta}\right)=(0,0),(0.2,0)$, $(-0.2,0)(0,0.2)$ and $(0,-0.2)$ respectively. The experimental data are from the compilation of Ref. [18].

\subsubsection{Point form kinematics}

The transition form factors calculated in point form with the same set of $D$-state components are shown in Figs. 6. The results are qualitatively similar to those obtained in instant form. The values for the transition magnetic moment form factor do however fall notably below those obtained with instant form kinematics at low values of momentum transfer, and hence further from the experimental values as well.

The values for $G_{E}^{*}$ are also correspondingly smaller, which on the other hand leads to a better description of the empirical values for $R_{E M}$ with the $D$-state amplitudes considered. The corresponding calculated values of the ratios $R_{E M}$ and $R_{S M}$ are shown in Fig. 7 . The introduction of the $D$-state component does not by itself capture the low momentum transfer peak of $R_{E M}$ with this form of kinematics. 

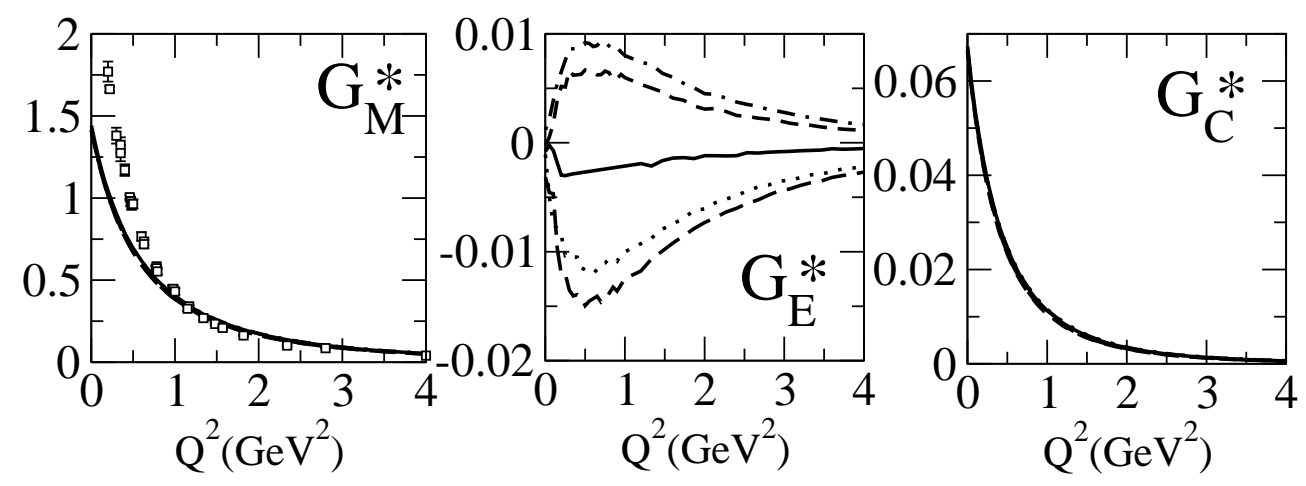

Fig. 6. $G_{E}^{*}, G_{M}^{*}$ and $G_{C}^{*}$ obtained in point form. Solid, dotted, dashed, long-dashed and dot-dashed lines stand for $\left(b_{N}, b_{\Delta}\right)=(0,0),(0.2,0),(-0.2,0)(0,0.2)$ and $(0,-0.2)$ respectively. In the case of $G_{M}^{*}$ and $G_{C}^{*}$ the lines are almost coincident. The data points for $G_{M}^{*}$ are from Ref. [17].

In the case of point form the introduction of $D$-state components also have but an insignificant effect on the calculated values of the Coulomb form factor $G_{C}^{*}$.
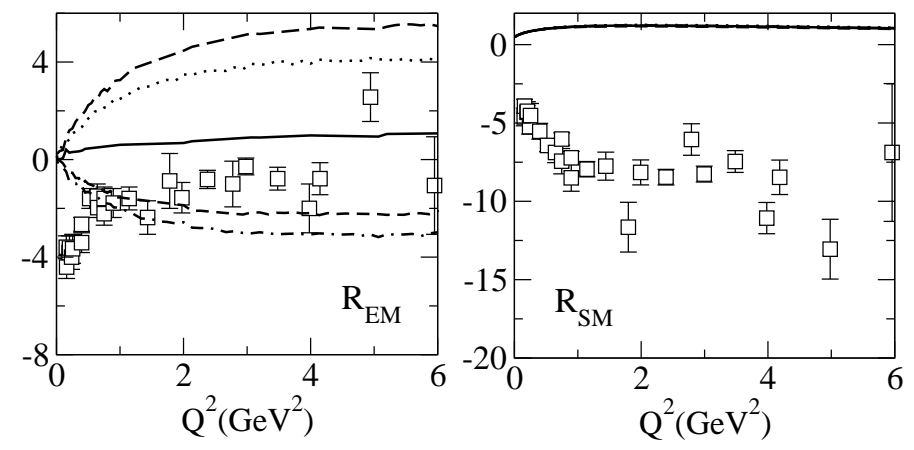

Fig. 7. $R_{E M}$ and $R_{S M}$ ratios in percent obtained in point form. Solid, dotted, dashed, long-dashed and dot-dashed lines stand for $\left(b_{N}, b_{\Delta}\right)=(0,0),(0.2,0),(-0.2,0)$ $(0,0.2)$ and $(0,-0.2)$ respectively. The experimental data are from the compilation of Ref. [18].

\subsubsection{Front form kinematics}

The transition form factors obtained in front form kinematics, in the reference frame where $Q^{+}=0$, are shown in Fig. 8. These form factors were calculated by using only the first three relations in Eq. (8). As noted above, the results depend on which set of spin amplitudes are employed [11].

The results for the magnetic transition form factor $G_{M}^{*}$ are similar to those obtained with instant form kinematics, and reveal very little sensitivity to the 

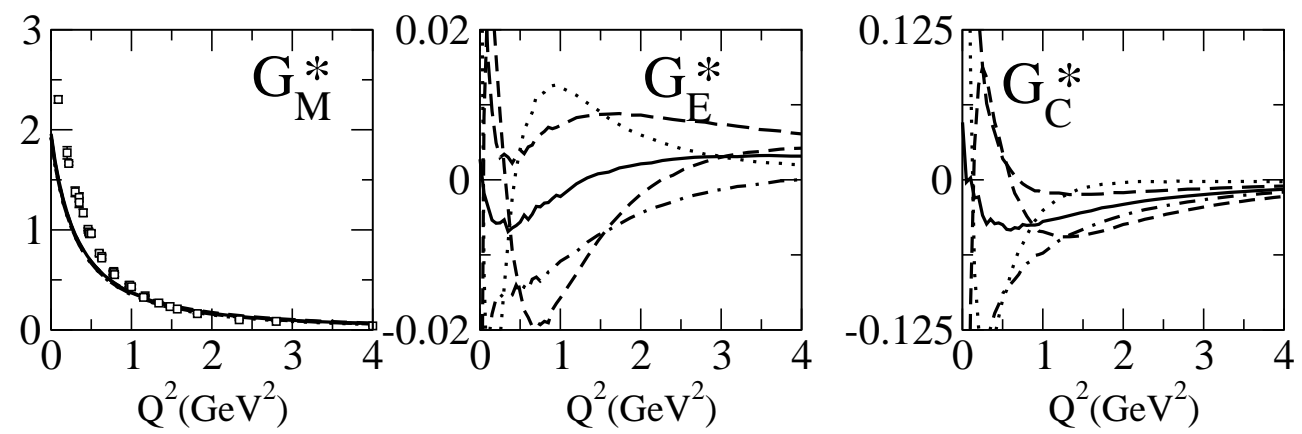

Fig. 8. $G_{E}^{*}, G_{M}^{*}$ and $G_{C}^{*}$ obtained in front form. The solid, dotted, dashed, long-dashed and dot-dashed lines stand for $\left(b_{N}, b_{\Delta}\right)=(0,0),(0.2,0),(-0.2,0)(0,0.2)$ and $(0,-0.2)$ respectively. In the case of $G_{M}^{*}$ and $G_{C}^{*}$ the lines are almost coincident. The data points for $G_{M}^{*}$ are from Ref. [17].

presence of $D$-state components in the nucleon and $\Delta(1232)$ wave function. The front form results for both the electric and Coulomb transition form factors are however very sensitive to the $D$-state components, as already noted in Ref. [11].

The corresponding results for the ratios $R_{E M}$ and $R_{S M}$ are shown in Fig. 9. The strong sensitivity to the $D$-state component is again evident from these figures. It is also found that the introduction of $D$-state components does not in general reduce the disagreement between the calculated and empirical values. For $R_{E M}$ the best description appears with a finite $D$-state component in the nucleon rather than in the $\Delta(1232)$ wave function.
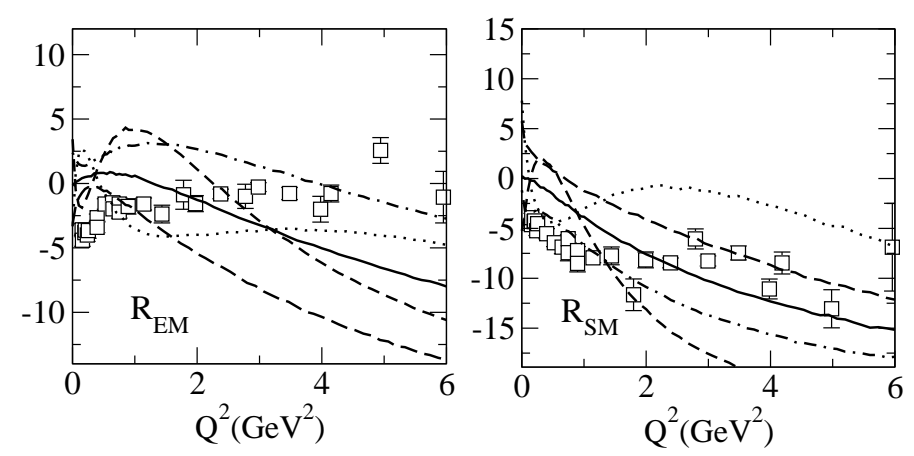

Fig. 9. $R_{E M}$ and $R_{S M}$ ratios in percent obtained in front form. Solid, dotted, dashed, long-dashed and dot-dashed lines stand for $\left(b_{N}, b_{\Delta}\right)=(0,0),(0.2,0),(-0.2,0)$ $(0,0.2)$ and $(0,-0.2)$ respectively. The experimental data are from the compilation of Ref. [18].

For transition form factors it should a priori be more natural to perform the calculation in the frame where $Q^{+} \neq 0$, as this allows smooth extrapolation to the timelike region. The transition form factors calculated in front form in this 

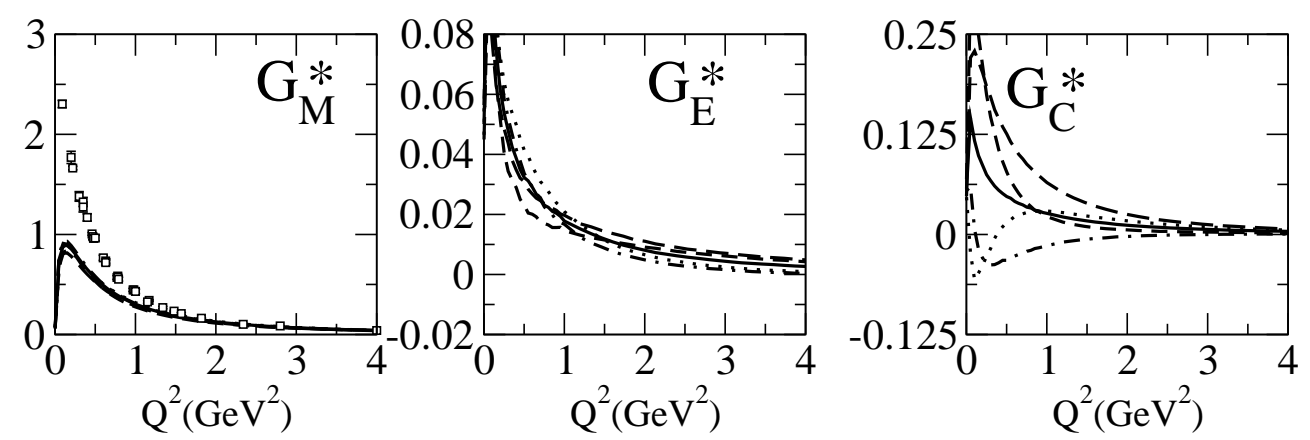

Fig. 10. $G_{E}^{*}, G_{M}^{*}$ and $G_{C}^{*}$ obtained in front form in the $Q^{+} \neq 0$ frame. Solid, dotted, dashed, long-dashed and dot-dashed lines stand for $\left(b_{N}, b_{\Delta}\right)=(0,0),(0.2,0)$, $(-0.2,0)(0,0.2)$ and $(0,-0.2)$ respectively. In the case of $G_{M}^{*}$ and $G_{C}^{*}$ the lines are almost coincident. The data points for $G_{M}^{*}$ are from Ref. [17].

frame are shown in Fig. 10. The results differ markedly from those obtained in the frame where $Q^{+}=0$. The magnetic transition form factor drops to 0 at $Q^{2}=0$, in disagreement with the empirical values. The calculated electric and Coulomb transition form factors also differ considerably from those obtained in the frame in which $Q^{+}=0$.

The zero in $G_{M}^{*}$ at $Q^{2}=0$ may be traced to the form of the relations (12) between the spin matrix elements and the invariant form factors $G_{j}$. If these equations are solved for the latter and the results are inserted in (4) it emerges that in this frame the transition form factors are linear combinations of the first three spin amplitudes with finite and nonvanishing coefficients in the limit $Q^{2} \rightarrow 0$. As all these spin amplitudes vanish with $Q^{2}$ it follows that all three transition form factors vanish with $Q^{2}$ as well.
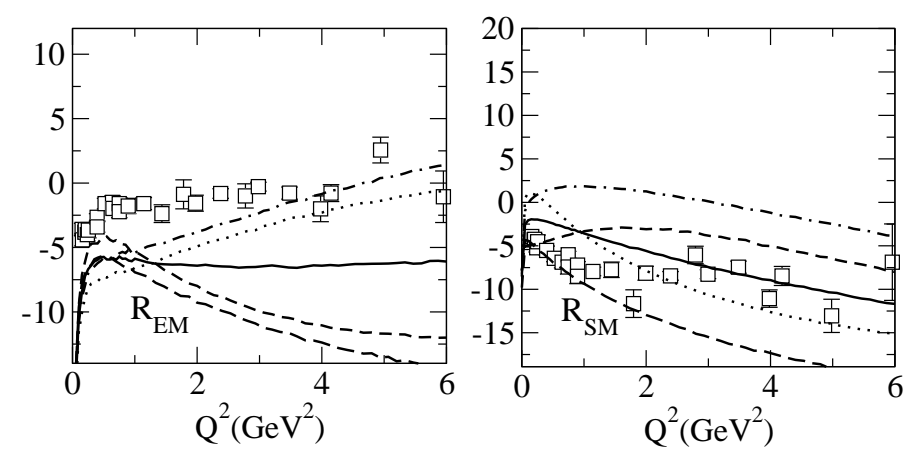

Fig. 11. $R_{E M}$ and $R_{S M}$ ratios in percent obtained in front form in the $Q^{+} \neq 0$ frame. Solid, dotted, dashed, long-dashed and dot-dashed lines stand for $\left(b_{N}, b_{\Delta}\right)=$ $(0,0),(0.2,0),(-0.2,0)(0,0.2)$ and $(0,-0.2)$ respectively. The experimental data are from the compilation of Ref. [18]. 
The ratios $R_{E M}$ and $R_{S M}$ that are obtained in front form kinematics in the frame $Q^{+} \neq 0$ are shown in Fig.11. They differ considerably from the corresponding results obtained in the frame in which $Q^{+}=0$. The ratio $R_{S M}$ in particular has a shape that deviates strongly from the empirical shape because of the small values of $G_{M}^{*}$ near $Q^{2}=0$.

\section{Conclusions}

Above a comprehensive study of the effect of $D$-state admixtures in the wave functions on electromagnetic form factors of the nucleon and the $\Delta(1232)-N$ transition form factors were carried out in three forms of relativistic kinematics with single constituent currents for pointlike quarks. The effects of the $D$-state admixtures on the elastic form factors of the proton and the magnetic form factor of the neutron were shown to be small. Only in the case of the electric form factor of the neutron is there a notable effect, although this was found to be smaller than that of a small mixed symmetry $S$-state component.

In instant and point form kinematics the effects of the $D$-state components on the magnetic and Coulomb $\Delta(1232)-N$ transition form factor were likewise found to be but minor. A notable sensitivity the $D$-state component was however found in the case of the electric $\Delta(1232)-N$ transition form factor. Consequently the ratio $R_{E M}$ is very sensitive to the $D$-state, while the ratio $R_{S M}$ is not.

In the case of front form kinematics the impulse approximation was found to violate the angular condition for the $\Delta(1232)-N$ spin matrix element to such a large extent that no useful predictions could be obtained for the electric and Coulomb transition form factors. This problem has also been noted in Ref. [11,12]. The calculated magnetic transition form factor is, however, similar to those obtained in front and instant form kinematics when calculated in the reference frame in which $Q^{+}=0$.

The empirically found structure in $R_{E M}$ at low momentum transfer could not be described by the inclusion of $D$-state deformation of the nucleon and $\Delta(1232)$ wave functions. Since this structure, if confirmed, represents a long range feature, and as it is well described in the coupled channel model of Sato and Lee [5], it is most likely due to a long range pionic fluctuation.

\section{Acknowledgment}

Instructive discussions with Drs. F. Coester and T.-S. H. Lee are acknowl-

edged. Research supported in part by the Academy of Finland through grant 
54038 and the European Euridice network HPRN-CT-2002-00311.

\section{References}

[1] G. A. Miller, Phys. Rev. C68 (2003) 022201.

[2] P. Jain and J. P. Ralston, hep-ph/0306194.

[3] A. M. Bernstein, Eur. Phys. J. A17 (2003) 249.

[4] R. Beck, Phys. Rev. Lett. 78 (1997) 606.

[5] T. Sato and T. S. H. Lee, Phys. Rev. C 54 (1996) 2660.

[6] T. Sato and T. S. H. Lee, Phys. Rev. C 63 (2001) 055201.

[7] P. A. M. Dirac, Rev. Mod. Phys. 49 (1949) 392.

[8] B. Juliá Díaz, D. O. Riska and F. Coester, Phys. Rev. C69 (2004) 035212.

[9] J. Bienkowska, Z. Dziembowski and H. J. Weber, Phys. Rev. Lett. 59 (1987) 624 [Erratum-ibid. 59 (1987) 1790].

[10] F. Coester, Prog. Part. Nucl. Phys. 29 (1992) 1.

[11] F. Cardarelli, E. Pace, G. Salme and S. Simula, Nucl. Phys. A 623 (1997) 361C;

[12] F. Cardarelli, E. Pace, G. Salme and S. Simula, Phys. Lett. B 371 (1996) 7; E. Pace, G. Salme, F. Cardarelli and S. Simula, Nucl. Phys. A 666 (2000) 33

[13] H. F. Jones and M. D. Scadron, Ann. Phys. 81 (1973) 1.

[14] R. C. E. Devenish, T. S. Eisenschitz and J. G. Korner, Phys. Rev. D 14 (1976) 3063.

[15] B. Juliá Díaz, D. O. Riska and F. Coester, nucl-th/0406015

[16] P. Mergell, U.-G. Meißner, D. Drechsel, Nucl. Phys. A 596, 367 (1996).

[17] W. W. Ash et al., Phys. Lett. B24 (1967) 165; W. Bartel et al., ibid, B28 (1968) 148; F. Foster and G. Hughes, Rep. Prog. Phys. 46 (1983) 1445; S. Stein et al., Phys. Rev. D12 (1975) 1884; V. V. Frolov et al., Phys. Rev. Lett. 82 (1999) 45; S. Galster et al., Phys. Rev. D5 (1972) 519

[18] V. D. Burkert and T. S. H. Lee, nucl-ex/0407020.

[19] C. E. Carlson, Phys. Rev. D 34 (1986) 2704.

[20] V.D. Burkert and L. Elouadrhiri, Phys. Rev. Lett. 75 (1995) 3614. 